LVESVI ( $p=0.01, r=-0.50$ ) after CRT. The further multivariate analysis showed only the plasma PIIINP level among clinic characters and all the biomarkers can predict the improvement of LVESV index $(\mathrm{OR}=8.33, P=0.01)$.

Conclusion The low PIIINP level, which is consistent with possible less cardiac fibrosis and a more plastic ventricle at baseline, is associated with CRT responsiveness. Contrary to previous reports, the NGF levels were not reduced during HF and that there was no NGF rebound in CRT responders.

\section{e0625 LEFT ATRIAL PRESSURE IS A DETERMINANT OF RECURRENCE IN ATRIAL FIBRILLATION AFTER CATHETER ABLATION}

doi:10.1136/hrt.2010.208967.625

${ }^{1}$ Ying-xue Dong, ${ }^{1}$ Yan-zong Yang, ${ }^{2,2}$ Jae K OhMasakiMitsuru, ${ }^{2}$ Brian D Powell, ${ }^{2}$ Mark D Larson, ${ }^{2}$ Traci L Buescher, ${ }^{2}$ David 0 Hodge, ${ }^{2}$ Douglas L Packer, ${ }^{1}$ Yong- Mei Cha. ${ }^{1}$ First Affiliated Hospital of Dalian Medical University, Dalian; ${ }^{2}$ The Department of Cardiology, The Division of Cardiovascular Diseases, Mayo Clinic, Rochester, Minnesota, USA

Introduction Pulmonary vein isolation is an effective therapy for curing symptomatic atrial fibrillation (AF). While it is known the severity of left atrial (LA) enlargement affect the success of AF ablation, little is known the impact of intracardiac pressure on the ablation outcome.

Methods This prospective study consisted of 63 patients (mean age $57 \pm 9$ years, $73 \%$ male) who underwent catheter-based pulmonary vein isolation for drug refractor symptomatic AF (48\% paroxysmal, $52 \%$ persistent). All patients underwent simultaneous echocardiography and haemodynamic measurements including left ventricular end systolic pressure (LVEDP), mean left atrial pressure (LAP) and $\mathrm{dP} / \mathrm{dt}_{\text {max }}$ using Millar catheter at the time of procedure during AF. Left atrial volume (LAV) was measure by biplane area length method. Recurrence of AF was defined as episodes of AF more than 5 min documented in $24 \mathrm{~h}$ ambulatory ECG or event monitor.

Results After a mean follow-up duration of $16 \pm 7$ months, AF elimination off anti-arrhythmic drugs was achieved in $70 \%(44 / 63)$ of patients. Among the echographic and haemodynamic measurements, the baseline LAV and mean LAP were $57.62 \pm 25.39 \mathrm{ml}$ and $12.19 \pm 4.57 \mathrm{~mm} \mathrm{Hg}$ in AF free patients compared to $81.20 \pm 40.88 \mathrm{ml}$ $(p=0.02)$ and $16.46 \pm 4.14 \mathrm{~mm} \mathrm{Hg}(p=0.01)$ in AF recurrence groups. Univariate and multivariate analysis showed LAP was the only independent predictor of the recurrence with an adjusted odd ratio of 1.27 ( $95 \%$ CI 1.04 to $1.54, \mathrm{p}=0.03$, table).

Conclusion LAP is a determinant of AF recurrence after AF ablation. Therapies towards reduction of LA filling pressure, especially in patients with elevated LAP, may improve the outcome of ablation.

Table Baseline clinical characters in the recurrence group and nonrecurrence group

\begin{tabular}{|c|c|c|c|c|c|c|}
\hline & \multirow{2}{*}{$\begin{array}{l}\text { Non-recurrence } \\
(\mathrm{N}=44)\end{array}$} & \multirow{2}{*}{$\begin{array}{l}\text { recurrence } \\
(N=19)\end{array}$} & \multicolumn{2}{|c|}{ Univariate } & \multicolumn{2}{|c|}{ Multivariate } \\
\hline & & & $\boldsymbol{P}$ & $95 \% \mathrm{Cl}$ & $\boldsymbol{P}$ & $95 \% \mathrm{Cl}$ \\
\hline Age (ys) & $57.39 \pm 9.92$ & $59.26 \pm 10.47$ & 0.49 & $0.97,1.08$ & - & - \\
\hline $\begin{array}{l}\text { AF type, persistent } \\
\text { AF no. }(\%)\end{array}$ & $23(53 \%)$ & $10(50 \%)$ & 0.97 & $0.34,2.98$ & - & - \\
\hline AF duration (ys) & $5.84 \pm 5.43$ & $8.08 \pm 7.66$ & 0.20 & $0.99,1.05$ & - & - \\
\hline LA volume $(\mathrm{mL})$ & $57.62 \pm 25.39$ & $81.20 \pm 40.88$ & 0.02 & $1.01,1.05$ & 0.30 & $0.99,1.05$ \\
\hline LVEDP (mm Hg) & $10.25 \pm 14.54$ & $11.25 \pm 5.75$ & 0.81 & $0.96,1.06$ & - & - \\
\hline $\mathrm{dP} / \mathrm{dt} \max (\mathrm{mm} \mathrm{Hg} / \mathrm{s})$ & $\begin{array}{l}1294.02 \pm \\
337.13\end{array}$ & $\begin{array}{l}1211.77 \pm \\
225.36\end{array}$ & 0.47 & $0.99,1.02$ & - & - \\
\hline Mean $\mathrm{LAP}_{\text {mean }}(\mathrm{mm} \mathrm{Hg})$ & $12.19 \pm 4.57$ & $16.46 \pm 4.14$ & 0.01 & $1.06,1.50$ & 0.02 & $1.04,1.54$ \\
\hline
\end{tabular}

Pulmonary vein isolation is an effective therapy for curing symptomatic atrial fibrillation (AF) but the success rate ranged wide. This study aimed to investigate the effect of LAP on AF recurrence.

\section{E0626 THE EFFECTS OF RECOMBINANT HUMAN B-TYPE NATRIURETIC PEPTID ON CORONARY CIRCULATION AND RENAL HAEMODYNAMICS IN YORK PIGS MODEL OF ACUTE MYOCARDIAL INFARCTION WITH HEART FAILURE}

doi:10.1136/hrt.2010.208967.626

Fu Xianghua, Zhang Jing, Wang Xuechao, Wang Yanbo, Xue Ling, Fan Weize, Wu Weili, Jiang Yunfa. The Second Hospital of Hebei Medical University. Shijiazhuang, Hebei China

Objective To evaluate the impact of intravenous administration of rhBNP on coronary and renal artery haemodynamics in York pigs model of AMI-ADHF.

Methods Fourteen York pigs were included in this study. After the AMI-ADHF models were established, pigs were randomised into saline group and rhBNP group. Coronary pressure $\left(\mathrm{P}_{\mathrm{c}}\right)$, the average peak velocity (APV), coronary vascular resistance (CR), coronary flow reserve (CFR) and coronary diameter were recorded simultaneously at baseline, instant after the model established, 60 min after continuous infusion of $0.01 \mu \mathrm{g} \cdot \mathrm{kg}^{-1} \cdot \mathrm{min}^{-1} \mathrm{rhBNP}$ and the time point of $\mathrm{LVEDP}<12 \mathrm{~mm} \mathrm{Hg}$. The blood flow of the coronary were measured at rest and maximal hyperaemia. Renal angiography was performed by $4 \mathrm{~F}$ catheter and quantitative measurement of diameter was recorded by the computer assisting system. The average peak rate of renal artery $\left(\mathrm{APV}_{\mathrm{ra}}\right)$ was recorded, determination of quantitative angiography of renal artery diameter, renal vascular resistance. LVEDP and LVEF was measured.

Results 1 . Coronary artery diameter increased after rhBNP administration. $\mathrm{APV}$ and $\mathrm{CBF}$ were significantly increased and $\mathrm{CR}$ decreased after rhBNP administration. CFR was significant rebound after continuous infusion of $0.01 \mu \mathrm{g} \cdot \mathrm{kg}^{-1} \cdot \mathrm{min}^{-1} \mathrm{rhBNP}$ for $30 \mathrm{~min}$. APV and CBF significantly increased and CR significantly decreased at the stage of infusion $0.010 \mu \mathrm{g} \mathrm{kg}^{-1} \mathrm{~min}^{-1} \mathrm{rhBNP}$ in rhBNP Group. 2. Renal artery pressure was significantly lower after rhBNP administration. RhBNP exerts renal vasodilator effects in a dose related relationship. RBF increased gradually after administration of rhBNP and was significantly higher than control group. RVR decreased after administration of rhBNP. LVEF was lower than baseline after the models established and tended to increase after administration of rhBNP.

Conclusion It could increase blood flow of injury coronary artery, improve CFR and improve the coronary and renal haemodynamics after intravenous administration of rhBNP in pigs with AMIADHF.

\section{e0627 THE PERIOPERATION EFFECT OF RECOMBINANT HUMAN B-TYPE NATRIURETIC PEPTIDE FOR HEART FAILURE PATIENTS WITH PRIMARY PERCUTANEOUS CORONARY INTERVENTION}

doi:10.1136/hrt.2010.208967.627

Fu Xianghua, Zhang Jing, Wang Xuechao, Wang Yanbo, Hao Guozhen, Fan Weize, Jiang Yunfa. The Second Hospital of Hebei Medical University. Shijiazhuang, Hebei China

Objective To study the efficacy and safety of recombinant human B-type natriuretic peptide (rhBNP) in AMI-ADHF patients undergoing PCI, especially changes in renal function and the impact of short-term outcome during BNP treatment.

Methods 87 consecutive patients with AMI-ADHF entrolled in the study. All patients were randomly assigned to the rhBNP group and control group. rhBNP was given at $1.5 \mu \mathrm{g} \mathrm{kg}^{-1}$ intravenously and then infused intravenously $\left(0.0075-0.030 \mu \mathrm{g} \mathrm{kg}{ }^{-1} \mathrm{~min}^{-1}\right) .0 .9 \%$ Saline was used intravenously in control group as control. Clinical symptoms and killip grade were recorded. Plasma BNP levels were measured before and after stopping the drug 6h, 14d, 30d. LVEDD 
and LVEF was measured. Serum creatinine (Scr) was measured before and after administered the medication 24 h, 48 h, 72 h, 7 days and 14 days using simplified MDRD equation to calculate estimated glomerular filtration rate (eGFR). Recording the major adverse cardiac events (MACE) occurrence within $30 \mathrm{~d}$.

Results rhBNP group has a less dyspnoea time than the control group; The plasma BNP levels significantly lower than before treatment at different time point in the two groups. The LVEF was significantly higher in treatment group compared with baseline levels after treatment $24 \mathrm{~h}$, while LVEDD significantly decreased even after discontinuation the treatments, which remain so when the 30 days. The LVEF and LVEDD improvements in rhBNP group were significantly better than in the control group after treatment 24 h, 14 days; At day 7 after PCI, the SCr had lowered to the baseline level in the rhBNP group. The eGFR after PCI was higher in the rhBNP group than that in the control group. The occurrence of CIN was signicantly lower in the rhBNP group than in the control group. The MACE event of 30 days in rhBNP group was significantly lower than the control group.

Conclusion rhBNP can promptly and effectively improve the heart function, reduce the incidence of MACE rate in acute myocardial infarction with heart failure patients, which also had a renal function protective effect in patients with and decreased incidence on CIN.

\section{e0628 CLINICAL ANALYSIS OF ACUTE MYOCARDIAL INFARCTION IN YOUNG PATIENTS}

doi:10.1136/hrt.2010.208967.628

Fu Xianghua, Wu Weili, Zhang Wenjing, Wang Xuechao, Wang Yanbo, Jiang Yunfa, Hao Guozhen. The Second Hospital of Hebei Medical University

Objective To investigate the clinical characteristics of acute myocardial infarction (AMI) in young patients.

Methods We carried out the contrasting analysis in the clinical data between 45 young patients (age $\leq 45$ years old) and 52 old patients (age $\geq 60$ years old).

Results Young AMI patients were often male, and had the typical clinical manifestations. The smoking rate, hyperfibrinogenemia rate and positive family history rate of the young people group were markedly higher than those of the old people group $(p<0.05)$. The morbidity rate of patients with single coronary artery atherosclerosis was high in the young people group. The morbidity rate of patients with multiple coronary artery atherosclerosis was high in the old people group. The patients in the old people group who complicated with cardiac aneurysm, arrhythmia, heart failure, cardiac shock were much more than those in the young people group $(p<0.05)$.

Conclusion Smoking, hyperfibrinogenemia and positive family history are main causes of AMI in young patients. Young AMI patients had the typical clinical manifestations with simple coronary lesion. The complications in the young people group are less than those in the old people group, and the prognosis was better than old cases.

\section{e0629 THE EFFECTS OF SLEEP APNOEA SYNDROME ON MYOCARDIAL ISCHAEMIA IN PATIENTS WITH CORONARY HEART DISEASE DURING NIGHT}

doi:10.1136/hrt.2010.208967.629

Fu Xianghua, Pang Jiangna, Wang Xuechao, Wang Yanbo, Jiang Yunfa, Wu Weili, Hao Guozhen, Gu Xinshun. The Second Hospital of Hebei Medical University

Objective 1. To investigate the relationship between sleep apnoea syndrome (SAS) and myocadial ischaemic events in patients with coronary heart disease (CHD). 2. To compare the differences of age, gender distribution, coronary angiography results in CHD patients with and without SAS. 3. To compare the differences of C-reactive protein (CRP) and haemoglobin levels in CHD patients with and without SAS.

Methods $25 \mathrm{CHD}$ patients with typical symptoms of angina and ECG changes were enrolled in this study. After overnight polysomnography (PSG), all the cases were monitored by portable device at night for 7 days in order to exclude the conditions that the cases did not sleep or had waked, apnoea and hypopnoea events were recorded during 24: 00-4:00. Blood samples were collected 5-10 min after monitoring, and the levels of haemoglobin and Creactive protein were examined.

Results 1 . The incidence of myocardial ischaemia caused by apnoea and low ventilation was significantly higher in CHD patients with SAS. 2. There were significant differences between the two groups in the decrease of oxygen desaturation and the increase of heart rate. 3 . BMI in CHD patients was significantly higher in those with SAS. There were more multi-vessel lesions and long lesions in CHD patients with SAS $(p<0.05)$. The level of haemoglobin and C-reactive protein were much higher in CHD patients with SAS.

Conclusion 1. The incidence of SAS is much higher in patients with $\mathrm{CHD}$, and the incidence of myocardial ischaemic events is higher in $\mathrm{CHD}$ patients with SAS. and the more seriuos respiratory disorders, the more easily myocardial ischaemia happens. With apnoea related to myocardial ischaemia and oxygen reduction, has nothing to do with the heart rate. 2. Lesions of SAS in patients with coronary heart disease are heavier than Simple CHD group in coronary angiography. BMI of SAS in patients with coronary heart disease are high than Simple CHD group. 3. The levels of CRP and haemoglobin are higher in CHD patients with SAS.

\section{e0630 THE INFLUENCE ON THE PLATELET FUNCTION OF DIFFERENT STATINS COMBINED WITH LOADING DOSE CLOPIDOGREL IN PATIENTS WITH ACUTE CORONARY SYNDROME}

doi:10.1136/hrt.2010.208967.630

Fu Xianghua, Wang Oian, Wang Yanbo, Wang Xuechao, Hao Guozhen, Jiang Yunfa, Fan Weize, Gu Xinshun. The Second Hospital of Hebei Medical University

Objective To investigate clinical effects of clopidogrel combined with simvastatin or fluvastatin on the platelet aggregation rate (PAR), platelet activation marker CD62P and the incidence of major adverse cardiovascular events (MACE) in patients with ACS.

Methods From April 2008 to December 2009, one hundred patients (79 male and 21 female, average age $61.46 \pm 12.84$ years) who had been diagnosed as ACS were enrolled into this study. These cases were randomly divided into two groups, the Group $A$ ( $n=50$, treated with simvastatin $20 \mathrm{mg}$ per night); the Group $B$ ( $n=50$, treated with fluvastatin $40 \mathrm{mg}$ per night). Detailed clinical information was collected. PAR, CD62P, alanine aminotransferase (ALT), and aspartate aminotransferase (AST) of the two groups were measured. All cases received clopidogrel (a loading dose of $300 \mathrm{mg}$ and then $75 \mathrm{mg}$ daily), aspirin and Low molecular weight heparin. The MACE within 14 days were recorded.

Results There was no significant differences in baseline between the Group A and Group B. There was no significant differences in the PAR and expression rate of CD62P after $300 \mathrm{mg}$ clopidogrel ( $p>0.05) .1 \mathrm{~h}$ after treated with statins the expression rate of CD62P and PAR in the two groups were lower than that before treated with statins $(p<0.05)$. After $14 d$ treated with statins the expression rate of CD62P and PAR were still lower than that before treated with statins $(p<0.05)$. There were no significant increase of ALT and AST in the both groups $(p>0.05)$. After the above-mentioned medical treatment, the expression rate of CD62P and PAR in the two groups 